\title{
DEGREE OF SYMMETRY OF A PRODUCT MANIFOLD
}

BY

\author{
H. T. KU, L. N. MANN, J. L. SICKS AND J. C. SU( $\left.{ }^{1}\right)$
}

0 . Introduction. Suppose $M^{m}$ is a compact connected differentiable $m$-manifold. Following [7], we define $N(M)$, the degree of symmetry of $M$, as the maximum of the dimensions of the isometry groups of all possible Riemannian structures on $M$. Of course, $N(M)$ is the maximum of the dimensions of the compact Lie groups which can act effectively and differentiably on $M$. It is well known that

$$
N\left(M^{m}\right) \leqq m(m+1) / 2,
$$

and that if $N\left(M^{m}\right)=m(m+1) / 2$, then $M$ is diffeomorphic to the standard sphere $S^{m}$ or the real projective space $R P^{m}$ [5]. In this paper we generalize this result by considering a product manifold

$$
M^{m}=M_{1}^{m_{1}} \times M_{2}^{m_{2}} \quad(m \geqq 19),
$$

where $M_{i}$ is a compact connected differentiable manifold of dimension $m_{i}$. We show that

and that if

$$
N(M) \leqq m_{1}\left(m_{1}+1\right) / 2+m_{2}\left(m_{2}+1\right) / 2
$$

$$
N(M)=m_{1}\left(m_{1}+1\right) / 2+m_{2}\left(m_{2}+1\right) / 2,
$$

then $M$ is a product of two spheres, two real projective spaces or one of each.

We establish the above result by first showing that if $M^{m}(m \geqq 19)$ is a compact connected differentiable $m$-manifold, then, except when $M$ is diffeomorphic to the complex projective space $C P^{k}(m=2 k)$,

$$
N\left(M^{m}\right) \leqq \alpha(\alpha+1) / 2+(m-\alpha)(m-\alpha+1) / 2,
$$

for all $\alpha$ such that $H^{\alpha}(M ; Q) \neq 0(Q=$ rationals $)$. This bound on $N\left(M^{m}\right)$ is of course sharper than $m(m+1) / 2$, especially if $\alpha$ can be chosen near [m/2]. It follows as an easy consequence of our results that

$$
N\left(C P^{k}\right)=k^{2}+2 k
$$

at least for $k \geqq 10$.

The authors are particularly indebted to Professor W. Y. Hsiang for several significant suggestions.

Received by the editors March 7, 1969.

( $\left.{ }^{1}\right)$ The second and fourth authors were supported in part by NSF GP-8929; the third author by NSF GP-8747. 
1. Preliminaries. Consider a transformation group $K$ acting on a space $X$. The subset $K_{0}$ of all elements of $K$ which act as the identity on $X$ form a normal subgroup of $K$, and $K / K_{0}$, in a natural fashion, acts effectively on $X$. The action of $K$ on $X$ is said to be almost effective if $K_{0}$ is finite. An almost effective action is said to be almost free if $K / K_{0}$ acts freely on $X$. The following lemma may be found in [11].

LEMMA 1. Let $K=K_{1} \times K_{2}$ act almost effectively on $X$. If $K_{1}$ acts transitively on $X$, then $K_{2}$ acts almost freely on $X$.

Following Jänich [9] we let $m(H)$, for a compact connected Lie group $H$, denote the minimal dimension of the connected manifolds upon which $H$ acts almost effectively, e.g.,

$$
m(\mathrm{SO}(n))=n-1 \text {. }
$$

The values of $m(H)$ for all compact simply-connected simple Lie groups $H$ are listed in $[9$, p. 68].

If a compact Lie group $G$ acts differentiably on a manifold $M$, it is known that the orbit space $M / G$ is a finite complex [15]. Furthermore it is well known that the dimension of $M / G$ is equal to the dimension of $M$ minus the dimension of a principal orbit.

A compact connected Lie group $G$ can be expressed in the form:

$$
G=\left(T^{q} \times S_{1} \times S_{2} \times \cdots \times S_{u}\right) / N=\bar{G} / N,
$$

where $T^{q}$ is a $q$-torus, $q \geqq 0$, each $S_{j}$ is a compact, connected, simply-connected simple Lie group and $N$ is a finite normal subgroup of $\bar{G}$. Each $S_{j}$ of dimension 3 is isomorphic to Spin (3). We employ the isomorphism

$$
\operatorname{Spin}(4) \cong \operatorname{Spin}(3) \times \operatorname{Spin}(3)
$$

to combine pairs of 3 -dimensional $S_{j}$ 's. With this convention, we may rewrite $G$ in the form

$$
G=\left(T^{q} \times S_{1}^{\prime} \times S_{2}^{\prime} \times \cdots \times S_{v}^{\prime}\right) / N=\bar{G} / N,
$$

where each $S_{j}^{\prime}$ is either simple or isomorphic to Spin (4) and where there is at most one $S_{j}^{\prime}$ of dimension 3 . We shall need later the following result [11, Theorem 1], $[9$, p. 68].

For a nonnegative integer $n$, let

$$
\langle n\rangle=n(n+1) / 2 \text {. }
$$

Proposition A. Let $G$ be a compact connected Lie group acting effectively on $a$ connected m-dimensional manifold $M$. If $G$ is of the form (B), then there exist integers $t_{1}, t_{2}, \ldots, t_{v}$ such that

$$
\operatorname{dim} S_{j}^{\prime} \leqq\left\langle t_{j}\right\rangle, \quad j=1,2, \ldots, v,
$$

and

$$
\sum_{j=1}^{v} t_{j} \leqq m-q .
$$


The following lemma is obvious.

LEMMA 2. If $n_{1} \geqq n_{2} \geqq w \geqq 0$,

(a) $\left\langle n_{1}\right\rangle+\left\langle n_{2}\right\rangle \leqq\left\langle n_{1}+n_{2}\right\rangle$,

(b) $\left\langle n_{1}\right\rangle+\left\langle n_{2}\right\rangle \leqq\left\langle n_{1}+w\right\rangle+\left\langle n_{2}-w\right\rangle$.

The next proposition is essentially due to W. Y. Hsiang [8] and related to Proposition A. The version presented here is easily derivable from $[9, \mathrm{p} .74]$.

Proposition B. Suppose $G$ acts effectively on a homogeneous space $G / H$ and

$$
\operatorname{dim} G>r(\operatorname{dim}(G / H)), \quad r \geqq 13 / 4 .
$$

Then if $G$ is of the form (B), there is at least one normal factor, say $S_{1}^{\prime}$, which is isomorphic to one of the following groups:

(i) Spin (n), $n>2 r$,

(ii) $\mathrm{SU}(n), n>2 r-1$,

(iii) $\mathrm{Sp}(n), n>2 r-2$.

REmarK. The restriction that $r \geqq 13 / 4$ in Proposition B guarantees that the dominant normal factor $S_{1}^{\prime}$ is a classical Lie group. If we remove this restriction, we obtain an expanded version of Proposition B which has exceptional simple Lie groups as possibilities for $S_{1}^{\prime}$.

\section{The main lemma.}

MaIN Lemma. Let $G$ be a compact connected Lie group acting differentiably and effectively on a compact connected m-manifold $M, m \geqq 19$. Then if

$$
\operatorname{dim} G \geqq m^{2} / 4+m / 2,
$$

exactly one of the following holds:

( $\alpha$ ) $M$ is diffeomorphic to $C P^{k}(m=2 k), G$ acts transitively on $M$ and $G$ is locally isomorphic to SU $(k+1)$.

( $\beta$ ) $M$ is diffeomorphic to $C P^{k} \times S^{1}(m=2 k+1), G$ acts transitively on $M$ and $G$ is locally isomorphic to $\mathrm{U}(k+1)$.

( $\gamma) M$ is a simple lens space finitely covered by $S^{2 k+1}(m=2 k+1), G$ acts transitively on $M$ and $G$ is locally isomorphic to $\mathrm{U}(k+1)$.

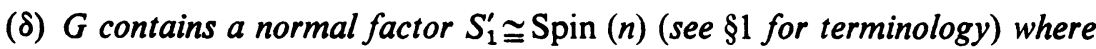

(a) $n \geqq m / 2+1$,

(b) $S_{1}^{\prime}$ acts almost effectively on $M$ with principal isotropy subgroup $H$ whose identity component $H^{0}$ is a standardly imbedded Spin $(n-1)$ in Spin $(n)$.

Proof. Let $G(x)$ be a principal orbit for the action of $G$ on $M$. Then $G$ acts effectively on the homogeneous space $G(x)=G / G_{x}$, where $G_{x}$ is the principal isotropy subgroup of $G$ at $x$. Now

$$
\operatorname{dim} G \geqq \frac{m^{2}}{4}+\frac{m}{2}=\left(\frac{m}{4}+\frac{1}{2}\right) m \geqq\left(\frac{m}{4}+\frac{1}{2}\right)\left(\operatorname{dim} \frac{G}{G_{x}}\right) .
$$


Let

$$
r=m / 4+1 / 2-\varepsilon,
$$

where $\varepsilon$ is a small positive quantity. Then

$$
\operatorname{dim} G>r \operatorname{dim}\left(G / G_{x}\right) \text {. }
$$

[Note that $r$ can be chosen equal to $m / 4+1 / 2$ if $\operatorname{dim} G(x)<m$.] Since $m \geqq 19$, it follows from (1) that $r>13 / 4$. Suppose now that $G$ is decomposed into the form (B). Then we may use (2) to apply Proposition B and obtain a normal factor group $S_{1}^{\prime}$ of $\bar{G}$ as described in the proposition. Since $\bar{G}$ acts almost effectively on $M$, so does $S_{1}^{\prime}$. We consider the possibilities for $S_{1}^{\prime}$.

Case I. $S_{1}^{\prime} \cong \mathrm{Sp}(n)$.

By Proposition B and (1),

Hence

$$
n>2 r-2=m / 2-1-2 \varepsilon .
$$

$$
n \geqq m / 2-1 \text {. }
$$

On the other hand,

$$
4 n-4=m(\operatorname{Sp}(n)) \leqq m
$$

or

$$
4 n \leqq m+4 .
$$

It follows from (3) and (4) that

$$
2 m-4 \leqq 4 n \leqq m+4
$$

or $m \leqq 8$. Hence Case $I$ is eliminated.

Case II. $S_{1}^{\prime} \cong \mathrm{SU}(n)$.

By Proposition B,

$$
n>2 r-1=m / 2-2 \varepsilon \text {. }
$$

Hence

$$
n \geqq m / 2 \text {. }
$$

Let $H$ denote a principal isotropy subgroup for the almost effective action of $S_{1}^{\prime}$ on $M$. We consider various cases for $\operatorname{dim} H$.

Subcase (a). $\operatorname{dim} H \leqq[\operatorname{dim} \mathrm{SU}(n-1)]-2$. Now,

$$
2 n+1 \leqq \operatorname{dim}\left(S_{1}^{\prime} / H\right) \leqq m .
$$

Hence

$$
2 n \leqq m-1 \text {. }
$$

We obtain an immediate contradiction from (5) and (6). Hence

$$
\operatorname{dim} H \geqq[\operatorname{dim} \mathrm{SU}(n-1)]-1 \text {. }
$$


On the other hand if $n \geqq 8$, which will always be the case from (5) if $m \geqq 15$, it follows automatically that

$$
\operatorname{dim} H \geqq \operatorname{dim} \mathrm{SU}(n-1) .
$$

(See, for example, [6, Theorem 1.19].)

Subcase (b). $\operatorname{dim} H=\operatorname{dim} \mathrm{SU}(n-1)$.

First let us show that $S_{1}^{\prime}$ is transitive on $M$. If not,

$$
2 n-1=\operatorname{dim}\left(S_{1}^{\prime} / H\right) \leqq m-1,
$$

or $2 n \leqq m$. It follows from (5) that $2 n=m$. Suppose $\bar{G}=S_{1}^{\prime} \times K$, where

$$
K=S_{2}^{\prime} \times \cdots \times S_{v}^{\prime} \times T^{q},
$$

in the decomposition (B). We shall show that $\operatorname{dim} K \leqq 3$, from which it follows that

$$
\operatorname{dim} G=\operatorname{dim} \bar{G}=\operatorname{dim} \mathrm{SU}(n)+\operatorname{dim} K \leqq n^{2}+2 .
$$

However since $m=2 n$,

$$
\operatorname{dim} G \leqq n^{2}+2<n^{2}+n=m^{2} / 4+m / 2,
$$

which contradicts our original assumption on the size of $\operatorname{dim} G$.

We proceed to show $\operatorname{dim} K \leqq 3$. Now $\bar{G}=S_{1}^{\prime} \times K$ acts transitively and almost effectively on a principal orbit $M_{1}=\bar{G}(x)$. Then $S_{1}^{\prime}$ acts transitively on the orbit space $M_{1} / K$ which must be a compact connected manifold. If $\operatorname{dim} K \geqq 4$, then $K$ acts on $M_{1}$ with principal orbits of dimension at least 3 and

$$
\operatorname{dim}\left(M_{1} / K\right) \leqq m-3=2 n-3 .
$$

Since $S_{1}^{\prime} \cong \mathrm{SU}(n)$ is simple, $S_{1}^{\prime}$ acts either almost effectively or trivially on $M_{1} / K$. However, since

$$
m(\mathrm{SU}(n))=2 n-2,
$$

the action must be trivial. Hence $M_{1} / K$ is a point and $K$ acts transitively on $M_{1}$. By Lemma $1, S_{1}^{\prime} \cong \mathrm{SU}(n)$ acts almost freely on $M_{1}$ and

$$
n^{2}-1=\operatorname{dim} \mathrm{SU}(n) \leqq m=2 n,
$$

which is an obvious contradiction. Hence $S_{1}^{\prime} \cong \mathrm{SU}(n)$ must act transitively on $M$. Since

$$
\operatorname{dim} H=\operatorname{dim} \operatorname{SU}(n-1),
$$

it follows, at least for $n \geqq 8$, that $H^{0}$, the identity component of $H$, is a standardly imbedded SU $(n-1)$ in SU (n) [6, Theorem 1.19]. We have the covering

$$
\begin{aligned}
& \tilde{M}=S_{1}^{\prime} / H^{0}=\mathrm{SU}(n) / \mathrm{SU}(n-1)=S^{2 n-1} \\
& \downarrow \\
& M=S_{1}^{\prime} / H
\end{aligned}
$$


and, therefore $M$ is finitely covered by $S^{2 n-1}$. Moreover the group of covering transformations $\mathrm{H} / \mathrm{H}^{0}$ is a subgroup of

$$
\frac{N(\mathrm{SU}(n-1), \mathrm{SU}(n))}{\mathrm{SU}(n-1)} \cong S^{1},
$$

where $N(\mathrm{SU}(n-1), \mathrm{SU}(n))$ denotes the normalizer of $\mathrm{SU}(n-1)$ in $\mathrm{SU}(n)$. Hence $H / H^{0}$ is a finite cyclic group and the action of $H / H^{0}$ on $\tilde{M}=S^{2 n-1}$ is the restriction of the standard free action of

$$
\frac{N(\mathrm{SU}(n-1), \mathrm{SU}(n))}{\mathrm{SU}(n-1)} \cong S^{1}
$$

on $S^{2 n-1}$. It follows that $M$ is a simple lens space. To show that we have possibility $(\gamma)$ of the Main Lemma we must now verify that $G$ is locally isomorphic to $\mathrm{U}(n)$. Recall that $\bar{G}=S_{1}^{\prime} \times K$. Now $S_{1}^{\prime} \cong \mathrm{SU}(n)$ acts transitively on $M$ and by Lemma 1 , $K$ acts almost freely on $M$. Furthermore, $S_{1}^{\prime}$ acts transitively on the compact connected manifold $M_{1}=M / K$. This action must also be nontrivial, and therefore almost effective, for otherwise $K$ would act transitively on $M$ and by Lemma 1 again, $S_{1}^{\prime}$ would act almost freely on $M$ which is impossible due to dimensional considerations. Now

and since

$$
\operatorname{dim} M_{1}=\operatorname{dim} M-\operatorname{dim} K=2 n-1-\operatorname{dim} K,
$$

$$
m(\mathrm{SU}(n))=2 n-2,
$$

it follows that $\operatorname{dim} K \leqq 1$. On the other hand,

$$
\operatorname{dim} \mathrm{SU}(n)=n^{2}-1=m^{2} / 4+m / 2-3 / 4,
$$

since $m=2 n-1$. It follows due to our dimensional restriction on $G$ that $\operatorname{dim} K=1$, and $K$ is a circle group. Since $\bar{G}=\mathrm{SU}(n) \times S^{1}, G$ is locally isomorphic to $\mathrm{U}(n)$ and we have possibility $(\gamma)$ of the Main Lemma.

Since $m(\mathrm{SU}(n))=2 n-2$, if $\operatorname{dim} H>\operatorname{dim} \mathrm{SU}(n-1)$, then we must have

$$
\operatorname{dim} H=\operatorname{dim} \mathrm{U}(n-1) .
$$

Subcase (c). $\operatorname{dim} H=\operatorname{dim} \mathrm{U}(n-1)$.

First we show that $S_{1}^{\prime}$ must act either transitively or with an $(m-1)$-dimensional orbit on $M$. If not,

$$
2 n-2=\operatorname{dim}\left(S_{1}^{\prime} / H\right) \leqq m-2,
$$

or $2 n \leqq m$. It follows from (5) that $2 n=m$, and we can proceed exactly as in Subcase (b) to obtain a contradiction.

It follows, at least for $n \geqq 8$, that $H^{0}$, the identity component of $H$, is a standardly imbedded $U$ for consistency $(n-1)$ in SU $(n)$. Since the normalizer of $U(n-1)$ in SU $(n)$ is $\mathrm{U}(n-1)$ itself, $H=H^{0}$. Suppose first that $S_{1}^{\prime}$ is transitive on $M$. Then

$$
M=\frac{S_{1}^{\prime}}{H}=\frac{\mathrm{SU}(n)}{\mathrm{U}(n-1)}=C P^{n-1} .
$$


As usual let $\bar{G}=S_{1}^{\prime} \times K$. Since $S_{1}^{\prime} \cong \mathrm{SU}(n)$ acts transitively on $M, K$ acts almost freely on $M$. Using our standard argument we may show $\operatorname{dim} K=0$, and, hence, that $G$ is locally isomorphic to $\mathrm{SU}(n)$. This gives us possibility $(\alpha)$ of the Main Lemma. By the way, in this case, since the Euler characteristic of $M=C P^{n-1}$ is positive, it follows directly that rank $K=0$.

We are left with the case that $S_{1}^{\prime}$ acts on $M$ with an $(m-1)$-dimensional orbit. It follows that

(i) $M / S_{1}^{\prime}=S^{1}$ or $I$ (closed interval),

(ii) All orbits are principal and diffeomorphic to $C P^{n-1}$.

(i) is a well-known result due to P. S. Mostert [13] and G. E. Bredon [3]. (ii) is reasoned out as follows. Since we have already agreed that the normalizer of $\mathrm{U}(n-1)$ in $\mathrm{SU}(n)$ is $\mathrm{U}(n-1)$ itself, all orbits of maximal dimension are principal and diffeomorphic to $C P^{n-1}$. Moreover since $H=\mathrm{U}(n-1)$, the only other possible orbits are fixed points. However, if the action has fixed points, then the orbit space is a closed interval with the two end points corresponding to the two exceptional orbits, each of which are fixed points. It follows that $M$ is homeomorphic to the suspension of $C P^{n-1}$ and therefore not a manifold since $n>2$.

Since all orbits are principal, $M$ is a fibre bundle over $M / S_{1}^{\prime}$ with structural group

$$
\frac{N[\mathrm{U}(n-1), \mathrm{SU}(n)]}{\mathrm{U}(n-1)},
$$

which is trivial. Hence $M=C P^{n-1} \times M / S_{1}^{\prime}$. Clearly $M / S_{1}^{\prime}=I$ is impossible. It follows that

$$
M=C P^{n-1} \times S^{1} .
$$

Let $\bar{G}=S_{1}^{\prime} \times K$ and suppose that $M_{1}=\bar{G}(x)$ is a principal orbit of the action of $\bar{G}$ on $M$. Since

$$
\operatorname{dim} M_{1} \leqq \operatorname{dim} M=2 n-1,
$$

and $S_{1}^{\prime} \cong \mathrm{SU}(n)$ acts almost effectively on $M_{2}=M_{1} / K$, it follows using our standard techniques that $\operatorname{dim} K \leqq 1$. On the other hand, due to our dimensional restriction on $G, \operatorname{dim} K=1$, and $G$ is locally isomorphic to $U(n)$. This gives us possibility $(\beta)$ of the Main Lemma. (Clearly $\bar{G}=\mathrm{SU}(n) \times S^{1}$ acts transitively on $M$.)

Case III. $S_{1}^{\prime} \cong \operatorname{Spin}(n)$.

By Proposition B,

Hence

$$
n>2 r=m / 2+1-2 \varepsilon .
$$

$$
n \geqq m / 2+1 \text {. }
$$

Let $H$ denote a principal isotropy subgroup of the almost effective action of $S_{1}^{\prime}$ on $M$. Suppose first that

$$
\operatorname{dim} H \leqq \operatorname{dim}[\mathrm{SO}(n-2) \times \operatorname{SO}(2)] .
$$


Now

$$
2 n-4 \leqq \operatorname{dim}\left(S_{1}^{\prime} / H\right) \leqq m,
$$

or

$$
2 n \leqq m+4
$$

Let $\bar{G}=S_{1}^{\prime} \times K$. We shall show that $\operatorname{dim} K \leqq m+6$, from which it will follow that

$$
\operatorname{dim} G=\operatorname{dim} \bar{G}=\operatorname{dim} \operatorname{Spin}(n)+\operatorname{dim} K \leqq n(n-1) / 2+m+6 .
$$

However, since from (8)

$$
\begin{aligned}
n & \leqq(m+4) / 2, \\
\operatorname{dim} G \leqq & \frac{\left(\frac{m+4}{2}\right)^{2}-\left(\frac{m+4}{2}\right)}{2}+m+6=\frac{m^{2}}{8}+\frac{7 m}{4}+7<\frac{m^{2}}{4}+\frac{m}{2},
\end{aligned}
$$

for $m \geqq 12$. This, of course, is a contradiction.

We proceed to show $\operatorname{dim} K \leqq m+6$. Now $\bar{G}=S_{1}^{\prime} \times K$ acts transitively and almost effectively on a principal orbit $M_{1}=\bar{G}(x)$. Hence $K$ acts transitively on the orbit space $M_{1} / S_{1}^{\prime}$ which must be a compact connected manifold. Let $M_{2}=M_{1} / S_{1}^{\prime}$. Now $S_{1}^{\prime}$ must act on $M$ with principal orbits of dimension at least $m-2$. For otherwise

$$
2 n-4 \leqq \operatorname{dim}\left(S_{1}^{\prime} / H\right) \leqq m-3,
$$

which contradicts (7). Hence

$$
\operatorname{dim} M_{2}=\operatorname{dim}\left(M_{1} / S_{1}^{\prime}\right) \leqq \operatorname{dim}\left(M / S_{1}^{\prime}\right) \leqq 2 .
$$

Now either $M_{2}$ is a point or $K$ acts nontrivially on $M_{2}$ since

$$
M_{2} / K=M_{1} / \bar{G}
$$

is a point. If $M_{2}$ is a point, $S_{1}^{\prime}$ is transitive on $M_{1}$ and by Lemma $1, K$ acts almost freely on $M_{1}$. Therefore $\operatorname{dim} K \leqq \operatorname{dim} M_{1} \leqq m$. Suppose then that $K$ acts nontrivially on $M_{2}$. Now

$$
K=S_{2}^{\prime} \times S_{3}^{\prime} \times \cdots \times S_{v}^{\prime} \times T^{q}
$$

and $1 \leqq \operatorname{dim} M_{2} \leqq 2$. Now either $T^{a}$ or $S_{j}^{\prime}, 2 \leqq j \leqq v$, acts nontrivially on $M_{2}$. Suppose, say, $S_{2}^{\prime}$ acts nontrivially on $M_{2}$. Then $S_{2}^{\prime}$ acts transitively on $M_{2}$ and $\operatorname{dim} S_{2}^{\prime} \leqq 6$. [Note that conceivably $S_{2}^{\prime} \cong \operatorname{Spin}(4)$.] Let

$$
K_{1}=S_{3}^{\prime} \times \cdots \times S_{v}^{\prime} \times T^{q} .
$$

Now $\bar{G}=S_{1}^{\prime} \times S_{2}^{\prime} \times K_{1}$, and $S_{1}^{\prime} \times S_{2}^{\prime}$ acts transitively on $M_{1}$. Hence by Lemma 1 , $K_{1}$ acts almost freely on $M_{1}$ and $\operatorname{dim} K_{1} \leqq \operatorname{dim} M_{1} \leqq m$. It follows that

$$
\operatorname{dim} K=\operatorname{dim} S_{2}^{\prime}+\operatorname{dim} K_{1} \leqq m+6 .
$$


If each $S_{j}^{\prime}, 2 \leqq j \leqq v$, acts trivially on $M_{2}$, then $T^{q}$ acts transitively and nontrivially on $M_{2}$. Therefore $T^{q}$ contains a subgroup $T^{2}$ which acts transitively on $M_{2}$. Let

$$
K_{2}=S_{2}^{\prime} \times S_{3}^{\prime} \times \cdots \times S_{v}^{\prime} \times T^{q-2}
$$

where $T^{q-2}=T^{q} / T^{2}$. Now $\bar{G}=S_{1}^{\prime} \times T^{2} \times K_{2}$ and $S_{1}^{\prime} \times T^{2}$ acts transitively on $M_{1}$. Hence by Lemma 1 again, $K_{2}$ acts almost freely on $M_{1}$ and

$$
\operatorname{dim} K=\operatorname{dim} T^{2}+\operatorname{dim} K_{2} \leqq 2+m .
$$

(Actually the above argument applies to the case where $\operatorname{dim} M_{2}=2 ;$ for $\operatorname{dim} M_{2}=1$, an obvious alteration is required.) It follows that

$$
\operatorname{dim} H>\operatorname{dim}[\mathrm{SO}(n-2) \times \mathrm{SO}(2)] \text {. }
$$

Hence if we assume $n \geqq 11$, which from (7) will always be the case for $m \geqq 19$, then $\operatorname{dim} H=\operatorname{dim} S O(n-1)$ and $H^{0}$ is a standardly imbedded Spin $(n-1)$ in Spin $(n)$ [6, Theorem 1.18]. Thus we have the last possibility $(\delta)$ of the Main Lemma.

\section{Main results.}

THEOREM 1. Let $M^{m}$ be a compact connected differentiable m-manifold ( $m \geqq 19$ ). Then precisely one of the following holds:

(i) $N\left(M^{m}\right) \leqq\langle\alpha\rangle+\langle m-\alpha\rangle$ for all $\alpha$ such that $H^{\alpha}(M ; Q) \neq 0$.

(ii) $M$ is diffeomorphic to $C P^{k}(m=2 k)$, and $N(M)=\operatorname{dim} \mathrm{SU}(k+1)$.

Proof. Suppose first that $M$ is diffeomorphic to $C P^{k}$. Now

$$
\begin{aligned}
H^{k}\left(C P^{k} ; Q\right) & \neq 0, & & k \text { even } ; \\
H^{k+1}\left(C P^{k} ; Q\right) & \neq 0, & & k \text { odd. }
\end{aligned}
$$

On the other hand SU $(k+1)$ acts almost effectively on $C P^{k}$. Consequently

$$
\begin{aligned}
N\left(C P^{k}\right) \geqq \operatorname{dim} \mathrm{SU}(k+1)=k^{2}+2 k & >\langle k\rangle+\langle k\rangle, & & k \text { even; } \\
& >\langle k+1\rangle+\langle k-1\rangle, & & k \text { odd. }
\end{aligned}
$$

We proceed to show that $C P^{k}$ is the only manifold which violates (i). Suppose $\left.N\left(M^{m}\right)\right\rangle\langle\alpha\rangle+\langle m-\alpha\rangle$, for some $\alpha$ where $H^{\alpha}(M ; Q) \neq 0$. Then there exists a compact connected Lie group $G$ acting effectively and differentiably on $M$ with

Now

$$
\left.N\left(M^{m}\right)=\operatorname{dim} G\right\rangle\langle\alpha\rangle+\langle m-\alpha\rangle .
$$

$$
\langle\alpha\rangle+\langle m-\alpha\rangle=\frac{\alpha^{2}+(m-\alpha)^{2}}{2}+\frac{m}{2} \geqq \frac{m^{2}}{4}+\frac{m}{2} .
$$

Hence $\operatorname{dim} G>m^{2} / 4+m / 2$, and we must be in one of the four possibilities of the Main Lemma. If possibility ( $\alpha$ ) occurs, $M$ is diffeomorphic to $C P^{k}$ and $G$ is locally isomorphic to $\mathrm{SU}(k+1)$. Hence $N(M)=\operatorname{dim} G=\operatorname{dim} \mathrm{SU}(k+1)$, and we have statement (ii). 
We show that the remaining possibilities of the Main Lemma cannot occur.

In the case of possibility $(\beta)$, we have

On the other hand,

$$
\operatorname{dim} G=\operatorname{dim} U(k+1)=(k+1)^{2} .
$$

$$
\langle\alpha\rangle+\langle m-\alpha\rangle \geqq\langle k+1\rangle+\langle k\rangle=(k+1)^{2} .
$$

Hence $\operatorname{dim} G \leqq\langle\alpha\rangle+\langle m-\alpha\rangle$.

If $M$ is finitely covered by $S^{m}$ (possibility $(\gamma)$ ), then $M$ is a rational cohomology sphere. (See, for example, [1, p. 38].) Hence $\alpha=0$ or $m$ and $\langle\alpha\rangle+\langle m-\alpha\rangle=\langle m\rangle$. However, $N\left(M^{m}\right) \leqq\langle m\rangle$ as is the case for every $m$-dimensional manifold. We are left with possibility $(\delta)$ of the Main Lemma. Now

$$
\bar{G}=S_{1}^{\prime} \times \cdots \times S_{v}^{\prime} \times T^{q}
$$

acts almost effectively on $M$ with

Hence

$$
S_{1}^{\prime} \cong \operatorname{Spin}(n), \quad n \geqq m / 2+1 .
$$

$$
\operatorname{dim} S_{1}^{\prime}=\operatorname{dim} \operatorname{Spin}(n)=\langle n-1\rangle \geqq\langle[(m+1) / 2]\rangle
$$

and by Proposition A,

$$
\operatorname{dim} S_{1}^{\prime} \geqq \operatorname{dim} S_{j}^{\prime}, \quad 2 \leqq j \leqq v .
$$

Let $\beta=\max (\alpha, m-\alpha)$. We claim it is sufficient to show that $n \leqq \beta+1$. For if

we know that

$$
\operatorname{dim} S_{1}^{\prime}=\operatorname{dim} \operatorname{Spin}(n)=\langle n-1\rangle \leqq\langle\beta\rangle,
$$

$$
\beta \geqq t_{1} \geqq t_{j}, \quad 2 \leqq j \leqq v
$$

from Proposition A. (Note we assume that the $t_{j}$ 's are chosen minimally.) Let

We know that

$$
t_{1}=\beta-u, \quad u \geqq 0 \text {. }
$$

$$
\operatorname{dim} G=\operatorname{dim} \bar{G} \leqq\langle\beta-u\rangle+\sum_{j=2}^{v}\left\langle t_{j}\right\rangle+q
$$

where

$$
\sum_{j=2}^{v} t_{j} \leqq m-q-(\beta-u)=m-\beta+u-q .
$$

We consider two cases.

(i) $\sum_{j=2}^{v} t_{j}+q \leqq u$. Then by Lemma 2 (a)

$$
\sum_{j=2}^{v}\left\langle t_{j}\right\rangle+q \leqq\left\langle\sum_{j=2}^{v} t_{j}+q\right\rangle \leqq\langle u\rangle,
$$

and from (9) it follows that

$$
\operatorname{dim} G=\operatorname{dim} \bar{G} \leqq\langle\beta-u\rangle+\langle u\rangle \leqq\langle\beta\rangle \leqq\langle\alpha\rangle+\langle m-\alpha\rangle .
$$


(ii) $\sum_{j=2}^{v} t_{j}+q>u$. By repeated use of Lemma 2 (b), since $\beta-u=t_{1} \geqq t_{j}$ for all $j$,

where

$$
\langle\beta-u\rangle+\sum_{j=2}^{v}\left\langle t_{j}\right\rangle+q \leqq\langle\beta\rangle+\sum_{j=2}^{v}\left\langle\tilde{t}_{j}\right\rangle+\tilde{q}
$$

(a) $0 \leqq \tilde{t}_{j} \leqq t_{j}$,

(b) $0 \leqq \tilde{q} \leqq q$,

(c) $\sum_{j=2}^{v} t_{j}+\tilde{q}=\sum_{j=2}^{v} t_{j}+q-u$.

From (9) it follows that

$$
\begin{aligned}
\operatorname{dim} G=\operatorname{dim} \bar{G} & \leqq\langle\beta\rangle+\sum_{j=2}^{v}\left\langle\tilde{t}_{j}\right\rangle+\tilde{q} \\
& \leqq\langle\beta\rangle+\left\langle\sum_{j=2}^{v} t_{j}+\tilde{q}\right\rangle \\
& \leqq\langle\beta\rangle+\left\langle\sum_{j=2}^{v} t_{j}+q-u\right\rangle \\
& \leqq\langle\beta\rangle+\langle m-\beta\rangle \quad \text { (from (10)) } \\
& \leqq\langle\alpha\rangle+\langle m-\alpha\rangle .
\end{aligned}
$$

It remains to show that $n \leqq \beta+1$. Suppose, on the contrary, that

$$
n \geqq \beta+2 \text {. }
$$

We know from the Main Lemma that $S_{1}^{\prime} \cong$ Spin $(n)$ acts almost effectively on $M$ with principal isotropy subgroup $H$ whose identity component $H^{0}$ is a standardly imbedded Spin $(n-1)$. It follows that the only possible orbits of the action of $S_{1}^{\prime}$ on $M$ are $S^{n-1}, R P^{n-1}$ and fixed points, all of which are acyclic over $Q$ up to and including dimension $n-2$.

Let $X$ denote the orbit space $M / S_{1}^{\prime}$. Then

$$
\operatorname{dim} X=m-(n-1) \leqq m-\beta-1<\alpha
$$

from (11). Consider the projection $\pi: M \rightarrow X$. Since $\pi^{-1}(x)$ is acyclic over $Q$ up to dimension $n-2$ for each $x$, it follows from the Vietoris-Begle Mapping Theorem [14] that

$$
H^{i}(M ; Q) \cong H^{i}(X ; Q), \quad i \leqq n-2 .
$$

However, since $\alpha \leqq \beta \leqq n-2$, it follows that $H^{\alpha}(X ; Q) \neq 0$ which contradicts (12). Hence $n \leqq \beta+1$ and we are finished.

THEOREM 2. Suppose

$$
M^{m}=M_{1}^{m_{1}} \times M_{2}^{m_{2}}, \quad\left(m_{1} \geqq m_{2} \geqq 1 ; m \geqq 19\right)
$$

where $M_{i}^{m_{1}}, i=1,2$, is a compact connected differentiable manifold of dimension $m_{i}$. Then $N\left(M^{m}\right) \leqq\left\langle m_{1}\right\rangle+\left\langle m_{2}\right\rangle$. Moreover if $N\left(M^{m}\right)=\left\langle m_{1}\right\rangle+\left\langle m_{2}\right\rangle$, then $M$ is diffeomorphic to one of the following:

(i) $S^{m_{1}} \times S^{m_{2}}$,

(ii) $R P^{m_{1}} \times R P^{m_{2}}$,

(iii) $S^{m_{1}} \times R P^{m_{2}}$,

(iv) $R P^{m_{1}} \times S^{m_{2}}$. 
Proof. For the first part of the theorem we may as well suppose that $M_{1}$ and $M_{2}$ are each orientable. If not, let

$$
\tilde{M}^{m}=\tilde{M}_{1}^{m_{1}} \times \tilde{M}_{2}^{m_{2}},
$$

where $\tilde{M}_{i}, i=1,2$ is either the orientable double covering of $M_{i}$ or $M_{i}$ itself depending upon whether or not $M_{i}$ is orientable. Now $\tilde{M}$ is either a two or four fold covering of $M$ and $N(\tilde{M}) \geqq N(M)$, since every group $G$ acting almost effectively on $M$ can be lifted to a covering group $\tilde{G}$ acting almost effectively on $\tilde{M}$. (See, for example, [10, Lemma 2].)

Now $H^{m_{1}}(M ; Q) \neq 0$, and since $C P^{k}$ does not split as the product of two manifolds, as is immediately obvious from its cohomology ring structure, it follows from Theorem 1 that $N\left(M^{m}\right) \leqq\left\langle m_{1}\right\rangle+\left\langle m_{2}\right\rangle$.

Suppose now that $N\left(M^{m}\right)=\left\langle m_{1}\right\rangle+\left\langle m_{2}\right\rangle$, and let $G$ be a compact connected Lie group of dimension $\left\langle m_{1}\right\rangle+\left\langle m_{2}\right\rangle$ acting effectively on $M$. If we refer to the Main Lemma, possibility $(\alpha)$ is out since $C P^{k}$ does not split. Possibility $(\gamma)$ is out for a similar reason. In possibility $(\beta)$ if

$$
C P^{k} \times S^{1}=M^{m_{1}} \times M^{m_{2}},
$$

then $m_{1}=2 k$ and $m_{2}=1$ due to the cohomology ring structure of $C P^{k} \times S^{1}$. However,

$$
\operatorname{dim} G=\operatorname{dim} U(k+1)\left\langle\langle 2 k\rangle+\langle 1\rangle=\left\langle m_{1}\right\rangle+\left\langle m_{2}\right\rangle .\right.
$$

Consequently we are dealing with possibility $(\delta)$ of the Main Lemma.

Let $\bar{G}=S_{1}^{\prime} \times K$. We know $S_{1}^{\prime} \cong \operatorname{Spin}(n)$, where $n \geqq m / 2+1$. Following the proof of Theorem 1 we can show that $n \leqq m_{1}+1$, lifting the action if necessary to the orientable covering $\tilde{M}=\tilde{M}_{1}^{m_{1}} \times \tilde{M}_{2}^{m_{2}}$. Let us first show that $n=m_{1}+1$. Suppose, on the contrary, that $n \leqq m_{1}$. Then $n-1 \leqq m_{1}-1$ and $\operatorname{dim} S_{1}^{\prime} \leqq\left\langle m_{1}-1\right\rangle$. Assume that $G$ is in the form (B) and apply Proposition A. Then

$$
\begin{aligned}
\operatorname{dim} G=\operatorname{dim} \bar{G} & =\operatorname{dim} S_{1}^{\prime}+\sum_{j=2}^{v} \operatorname{dim} S_{j}^{\prime}+q \\
& \leqq\left\langle t_{1}\right\rangle+\sum_{j=2}^{v}\left\langle t_{j}\right\rangle+q .
\end{aligned}
$$

We know

$$
t_{j} \leqq t_{1} \leqq m_{1}-1, \quad j \geqq 2 .
$$

If $m_{2} \leqq m_{1}-2$, we may use Lemma 2 to show

$$
\operatorname{dim} G \leqq\left\langle m_{1}-1\right\rangle+\left\langle m_{2}+1\right\rangle\left\langle\left\langle m_{1}\right\rangle+\left\langle m_{2}\right\rangle .\right.
$$

If $m_{2}=m_{1}-1$ we can conclude by Lemma 2 that

$$
\operatorname{dim} G \leqq\left\langle m_{1}-1\right\rangle+\left\langle m_{2}\right\rangle+\langle 1\rangle\left\langle\left\langle m_{1}\right\rangle+\left\langle m_{2}\right\rangle .\right.
$$

Finally, if $m_{2}=m_{1}$,

$$
\operatorname{dim} G \leqq\left\langle m_{1}-1\right\rangle+\left\langle m_{2}-1\right\rangle+\langle 2\rangle\left\langle\left\langle m_{1}\right\rangle+\left\langle m_{2}\right\rangle .\right.
$$

We proceed with $n=m_{1}+1$. 
Choose $x \in M$ so that it lies simultaneously on principal orbits of the actions of $S_{1}^{\prime}, K$ and $\bar{G}$ on $M$, and let $H_{1}, H_{2}$ and $H$, respectively, denote the isotropy subgroups at $x$ of the three actions. Then $N_{1}=\bar{G}(x)$ is a principal orbit for the action of $\bar{G}$ on $M$ and $\bar{G}=S_{1}^{\prime} \times K$ acts transitively and almost effectively on $N_{1}$. We know that

$$
\operatorname{dim} K=\operatorname{dim} \bar{G}-\operatorname{dim} S_{1}^{\prime}=\left\langle m_{2}\right\rangle,
$$

and therefore that $K$ acts almost effectively on $N_{1}$ with principal orbits of dimension at least $m_{2}$. On the other hand, if the dimension of the principal orbits of this action is greater than $m_{2}$, it follows that

$$
\operatorname{dim} N_{2}<m-m_{2}=m_{1},
$$

where $N_{2}$ denotes the orbit space $N_{1} / K$. However $S_{1}^{\prime}$, which is simple, acts either trivially or almost effectively on the compact connected manifold $N_{2}$. If the action is trivial, then $N_{2}$ is a point and, therefore, $K$ acts transitively on $N_{1}$. It then follows from Lemma 1 that $S_{1}^{\prime}$ acts almost freely on $N_{1}$ and that

$$
\left\langle m_{1}\right\rangle=\operatorname{dim} S_{1}^{\prime} \leqq \operatorname{dim} N_{1} \leqq m,
$$

which is impossible. But if $S_{1}^{\prime}$ acts almost effectively on $N_{2}$ we again arrive at a contradiction since $\operatorname{dim} S_{1}^{\prime}=\left\langle m_{1}\right\rangle$ and $\operatorname{dim} N_{2}<m_{1}$. Therefore $K$ acts on $N_{1}$ with principal orbits precisely of dimension $m_{2}$. It follows that $K \cong \operatorname{Spin}\left(m_{2}+1\right)$, and that

$$
\operatorname{dim} \bar{G}(x)=\operatorname{dim} N_{1}=\operatorname{dim} N_{2}+m_{2}=m_{1}+m_{2}=m .
$$

Hence $\bar{G}$ acts transitively on $M$ and $M=\bar{G} / H$. We already know that $H_{1}^{0}$ is a standardly imbedded Spin $\left(m_{1}\right)$ in $S_{1}^{\prime}$. From our knowledge of the action of $K$ on $N_{1}=M$, it follows that

$$
\operatorname{dim} H_{2}^{0}=\operatorname{dim} \operatorname{Spin}\left(m_{2}\right),
$$

and consequently for $m_{2} \neq 3,7$ that $H_{2}^{0}$ is a standardly imbedded Spin $\left(m_{2}\right)$ in $K$ [12, Lemma 7]. We proceed under the assumption that $m_{2} \neq 3,7$ acknowledging that separate arguments are required to handle these two possibilities. In any case $H_{1}^{0} \times H_{2}^{0} \subset H^{0}$, and since it is immediately checked that $\operatorname{dim}\left(H_{1}^{0} \times H_{2}^{0}\right)=\operatorname{dim} H^{0}$, it follows that $H_{1}^{0} \times H_{2}^{0}=H^{0}$.

Now $H / H^{0} \subset N\left(H^{0}, \bar{G}\right) / H^{0}$ and

$$
\begin{aligned}
\frac{N\left(H^{0}, \bar{G}\right)}{H^{0}} & =\frac{N\left(\operatorname{Spin}\left(m_{1}\right), \operatorname{Spin}\left(m_{1}+1\right)\right)}{\operatorname{Spin}\left(m_{1}\right)} \times \frac{N\left(\operatorname{Spin}\left(m_{2}\right), \operatorname{Spin}\left(m_{2}+1\right)\right)}{\operatorname{Spin}\left(m_{2}\right)} \\
& \cong Z_{2} \oplus Z_{2} .
\end{aligned}
$$

We obtain the possibilities (i), (ii), (iii) and (iv) listed in Theorem 2 by taking $H / H^{0}$ to be the subgroups $0, Z_{2} \oplus Z_{2}, 0 \oplus Z_{2}$ and $Z_{2} \oplus 0$ respectively. The only remaining possibility is that $H / H^{0}$ is a $Z_{2}$ imbedded diagonally in $Z_{2} \oplus Z_{2}$. In this case $M$ can be described as follows. Let $Z_{2}$ act on $S^{m_{1}} \times S^{m_{2}}$ by $(x, y) \rightarrow$ $(-x,-y)$. Then

$$
M=\left(S^{m_{1}} \times S^{m_{2}}\right) / Z_{2}
$$


is the orbit space of this action. We shall show that, depending upon $m_{1}$ and $m_{2}$, $M$ may or may not split as a product but when $M$ does split, then it is diffeomorphic to $S^{m_{1}} \times R P^{m_{2}}$. This would complete the proof of Theorem 2 .

Recall [2] that $(K O) \sim\left(R P^{m_{2}}\right)$ is a cyclic group of order $2^{\phi\left(m_{2}\right)}$ where

such that

$$
\phi\left(m_{2}\right)=\text { the number of integers } p
$$

$$
\begin{gathered}
0<p \leqq m_{2} \\
p \equiv 0,1,2,4 \quad(\bmod 8) .
\end{gathered}
$$

Moreover a generator of $(K O) \sim\left(R P^{m_{2}}\right)$ is given by the element $\alpha=\xi-1$, where $\xi$ is the usual Hopf bundle

$$
\xi: Z_{2} \rightarrow S^{m_{2}} \rightarrow R P^{m_{2}}
$$

defined by the antipodal involution of $Z_{2}$ on $S^{m_{2}}$. We complete the proof of Theorem 2 by proving the following proposition.

Proposition C. Let

$$
M=\left(S^{m_{1}} \times S^{m_{2}}\right) / Z_{2}
$$

be the orbit space of the diagonal involution $(x, y) \rightarrow(-x,-y)$ on $S^{m_{1}} \times S^{m_{2}}$, $m_{1} \geqq m_{2}$. Then $M$ splits as a product if and only if

$$
m_{1}+1 \equiv 0 \quad\left(\bmod 2^{\phi\left(m_{2}\right)}\right) .
$$

Moreover, if $M$ splits, then it is diffeomorphic to $S^{m_{1}} \times R P^{m_{2}}$.

Proof. First observe that we have the fibration

$$
\eta: S^{m_{1}} \rightarrow M \rightarrow R P^{m_{2}},
$$

which is the bundle associated with $\xi$ having fiber $S^{m_{1}}$. Thus as vector bundles, $\eta=\left(m_{1}+1\right) \xi$. If

then

$$
m_{1}+1 \equiv 0 \quad\left(\bmod 2^{\phi\left(m_{2}\right)}\right),
$$

$$
\eta-\left(m_{1}+1\right)=\left(m_{1}+1\right) \alpha=0
$$

in $(K O) \sim\left(R P^{m_{2}}\right)$. This means that $\eta$ is stably trivial. But since $m_{2}<m_{1}+1$, we are in the stable range and therefore $\eta$ itself is a trivial bundle. In particular, $M$ is diffeomorphic to $S^{m_{1}} \times R P^{m_{2}}$.

Suppose now that $M=M_{1}^{m_{1}} \times M_{2}^{m_{2}}$. We wish to show that

$$
m_{1}+1 \equiv 0 \quad\left(\bmod 2^{\phi\left(m_{2}\right)}\right) .
$$

We shall consider only the case $m_{2}>1$ as the argument for $m_{2}=1$ is similar. If $m_{2}>1$, then $\pi_{1}(M)=Z_{2}$, and hence one of the two factors $M_{1}$ and $M_{2}$ is simply connected. Denote the simply connected factor by $M_{\beta}$ and the other by $M_{r}$. Then $\pi_{1}\left(M_{\gamma}\right)=Z_{2}$. Let $\tilde{M}_{y}$ be the universal covering space of $M_{\gamma}$. Then we must have

$$
M_{\beta} \times \tilde{M}_{y} \approx S^{m_{1}} \times S^{m_{2}}
$$


From this it follows that $M_{\beta}$ and $\tilde{M}_{y}$ must be homotopy spheres of dimensions $m_{1}$ or $m_{2}$. If $m_{1}=m_{2}$, it does not matter of course which is which. But if $m_{1}>m_{2}$, we claim that

$$
\operatorname{dim} M_{\beta}=m_{1}, \quad \operatorname{dim} M_{y}=m_{2} .
$$

For if it were the reverse way, then $M$ would have the mod 2 cohomology

$$
H^{*}\left(M ; Z_{2}\right)=H^{*}\left(R P^{m_{1}} \times S^{m_{2}} ; Z_{2}\right) \text {. }
$$

However, a simple spectral sequence computation (the spectral sequence is trivial because $m_{1}>m_{2}$ ) shows that

$$
H^{*}\left(M ; Z_{2}\right)=H^{*}\left(S^{m_{1}} \times R P^{m_{2}} ; Z_{2}\right),
$$

at least additively. But this gives an immediate contradiction by looking at $H^{m_{2}}\left(M ; Z_{2}\right)$.

Thus at any rate $M$ is homotopy equivalent to

$$
S^{m_{1}} \times R P^{m_{2}} \text {. }
$$

Let

$$
h: M \rightarrow S^{m_{1}} \times R P^{m_{2}}
$$

be a homotopy equivalence and let

$$
i: S^{m_{1}} \rightarrow M
$$

be the inclusion of a fiber in $\eta$. It is easily checked that

$$
S^{m_{1}} \stackrel{i}{\longrightarrow} M \stackrel{h}{\longrightarrow} S^{m_{1}} \times R P^{m_{2}} \stackrel{\rho_{1}}{\longrightarrow} S^{m_{1}}
$$

induces an isomorphism on the integral cohomology in dimension $m_{1}$. According to Dold [4], this means the bundle $\eta$ is fiber homotopically trivial. However, it is known [2] that the $J$-homomorphism

$$
J:(K O)^{\sim}\left(R P^{m_{2}}\right) \rightarrow J\left(R P^{m_{2}}\right)
$$

is an isomorphism for projective spaces. Hence the element

$$
\eta-\left(m_{1}+1\right)=\left(m_{1}+1\right)(\xi-1)=\left(m_{1}+1\right) \alpha
$$

is zero in $(K O) \sim\left(R P^{m_{2}}\right)$ or

$$
m_{1}+1 \equiv 0 \quad\left(\bmod 2^{\phi\left(m_{2}\right)}\right) .
$$

REMARKS. The assumption that $m \geqq 19$ throughout the paper appears to be technical and we are able to dispose of this restriction in most cases. The authors are currently attempting to generalize Theorem 2 to products of more than two manifolds. The key to the situation appears to involve increasing the scope of the Main Lemma. For example, to handle the product of three manifolds, we should consider manifolds $M$ where

$$
N(M) \geqq m^{2} / 6+m / 2 ;
$$


for the product of four manifolds, we should consider manifolds $M$ where

$$
N(M) \geqq m^{2} / 8+m / 2
$$

and so on.

It is easily checked that differentiability is not really used in the proof of the Main Lemma. Consequently, Theorems 1 and 2 can be stated in terms of topological manifolds and topological degree of symmetry $N_{T}\left(M^{m}\right)$ where for a compact connected topological $m$-manifold $M^{m}, N_{Y}\left(M^{m}\right)$ is the maximum of the dimensions of the compact Lie groups which can act effectively on $M$.

Proposition D. Suppose

$$
M=M_{1} \times M_{2} \times \cdots \times M_{\lambda},
$$

where $M$ and the $M_{i}$ are compact differentiable manifolds. Then

$$
N(M) \geqq \sum_{i=1}^{\lambda} N\left(M_{i}\right)
$$

Proof. Let $\tilde{M}_{i}$ be a Riemannian structure for $M_{i}$ such that

$$
N\left(M_{i}\right)=\operatorname{dim} \operatorname{Isom}\left(\tilde{M}_{i}\right), \quad i=1,2, \ldots, \lambda .
$$

Let $\tilde{M}=\tilde{M}_{1} \times \tilde{M}_{2} \times \cdots \times \tilde{M}_{\lambda}$ be the product Riemannian manifold. Clearly Isom $(\tilde{M})$ contains a subgroup isomorphic to the product

Hence

$$
\operatorname{Isom}\left(\tilde{M}_{1}\right) \times \operatorname{Isom}\left(\tilde{M}_{2}\right) \times \cdots \times \operatorname{Isom}\left(\tilde{M}_{\lambda}\right) \text {. }
$$

$$
N(M) \geqq \operatorname{dim} \operatorname{Isom}(\tilde{M}) \geqq \sum_{i=1}^{\lambda} N\left(M_{\mathfrak{i}}\right) .
$$

EXAMPLE. If $\Sigma^{n}$ is an exotic sphere which bounds a $\pi$-manifold, it is known that $\Sigma^{n} \times S^{2}$ is diffeomorphic to $S^{n} \times S^{2}$. Furthermore, it is known [7] that

It follows that

$$
N\left(\Sigma^{n}\right)<\frac{1}{8} n^{2}+1 .
$$

$$
N\left(\Sigma^{n} \times S^{2}\right)>N\left(\Sigma^{n}\right)+N\left(S^{2}\right) .
$$

Corollary 1 (Corollary to Theorem 2 ).

$$
N\left(M_{1}\right)+N\left(M_{2}\right) \leqq N\left(M_{1}^{m_{1}} \times M_{2}^{m_{2}}\right) \leqq\left\langle m_{1}\right\rangle+\left\langle m_{2}\right\rangle \quad\left(m_{1}+m_{2} \geqq 19\right) .
$$

REMARK. If the right-hand equality in the above corollary holds we know that $M_{1} \times M_{2}$ is diffeomorphic to one of the four manifolds listed in Theorem 2. However, this does not imply that the left-hand equality holds as exhibited by the last example where we chose an exotic splitting $M_{1}=\Sigma^{n}, M_{2}=S^{2}$ of $S^{n} \times S^{2}$.

\section{REFERENCES}

1. A. Borel et al., Seminar on transformation groups, Ann. of Math. Studies, Princeton Univ. Press, Princeton, N. J., 1960.

2. R. Bott, Lectures on $K(X)$, Harvard Univ., Cambridge, Mass., 1963.

3. G. E. Bredon, Some theorems on transformation groups, Ann. of Math. 67 (1958), 104-118. 
4. A. Dold, Uber fasernweise Homotopieäquivalenz von Faserräumen, Math. Z. 62 (1955), 111-136.

5. L. P. Eisenhart, Riemannian geometry, Princeton Univ. Press, Princeton, N. J., 1949.

6. W. C. Hsiang and W. Y. Hsiang, Differentiable actions of compact connected classical groups. I, Amer. J. Math. 89 (1967), 705-786.

7. W. Y. Hsiang, On the bound of the dimensions of the isometry groups of all possible Riemannian metrics on an exotic sphere, Ann. of Math. 85 (1967), 351-358.

8. - The natural metric on $\mathrm{SO}(n) / \mathrm{SO}(n-2)$ is the most symmetric metric, Bull. Amer. Math. Soc. 73 (1967), 55-58.

9. K. Jänich, Differenzierbare G-Mannigfaltigkeiten, Springer-Verlag, Berlin, 1968.

10. J. M. Kister and L. N. Mann, Isotropy structure of compact Lie groups on complexes, Michigan Math. J. 9 (1962), 93-96.

11. L. N. Mann, Gaps in the dimensions of transformation groups, Illinois J. Math. 10 (1966), 532-546.

12. Deane Montgomery and Hans Samelson, Transformation groups of spheres, Ann. of Math. 44 (1943), 454-470.

13. Paul S. Mostert, On a compact Lie group acting on a manifold, Ann. of Math. 65 (1957), 447-455.

14. E. H. Spanier, Algebraic topology, McGraw-Hill, New York, 1966.

15. C. T. Yang, The triangulability of the orbit space of a differentiable transformation group, Bull. Amer. Math. Soc. 69 (1963), 405-408.

UNIVERSTTY OF MASSACHUSETTS, AmHerst, MassachusetTS 